\title{
Pregnancy Complicated with Acute Promyelocytic Leukemia: A Case Report and a Literature Review
}

\author{
Ying Li, Pingyong Li*, Juanli He, Qiongjin Li \\ First Affiliated Hospital of Dali University, Dali, China \\ Email: ^2573040621@qq.com
}

How to cite this paper: Li, Y., Li, P.Y., He, J.L. and Li, Q.J. (2020) Pregnancy Complicated with Acute Promyelocytic Leukemia: A Case Report and a Literature Review. Yangtze Medicine, 4, 163-172. https://doi.org/10.4236/ym.2020.43016

Received: October 31, 2019

Accepted: June 27, 2020

Published: June 30, 2020

Copyright ( 2020 by author(s) and Scientific Research Publishing Inc. This work is licensed under the Creative Commons Attribution International License (CC BY 4.0).

http://creativecommons.org/licenses/by/4.0/ (c) (i) Open Access

\begin{abstract}
Acute promyelocytic leukemia (APL) is a special subgroup of acute myeloid leukemia (AML). About $95 \%$ of the patients have specific chromosome translocation $\mathrm{t}(15 \leq 17)$ ( $\mathrm{q} 22, \mathrm{q} 12)$. APL progresses rapidly and is characterized by extensive and severe bleeding and disseminated intravascular coagulation (DIC). Patients may present with severe clinical manifestation which is often caused by the occurrence of DIC in the early stages of the disease. Pregnancy complicated with APL is rare in the clinical setting. They have many complications, high mortality and are difficult to manage clinically. This case report describes a case of a primary pregnancy complicated with APL, in order to better understand how to manage such complex cases.
\end{abstract}

\section{Keywords}

Gestational APL, ATRA, Leukemia, Bleeding

\section{Introduction}

Acute promyelocytic leukemia is a special subtype of acute myeloid leukemia, accounting for about $10 \%$ of AML. Pregnancy combined with APL is clinically rare. It has been reported in the literature, its incidence is about 1 in 75,000 to 1 in 100,000 pregnant women [1] [2]. Combined with acute blood cell abnormalities and coagulation dysfunction, acute promyelocytic leukemia is extremely harmful to mothers and children. On the one hand, it can cause abortion, premature delivery, intrauterine growth restriction, fetal and maternal death. On the other hand, its treatment is very challenging for clinicians. Chemotherapy is controversial during pregnancy due to its potential teratogenic risks. In addition, complications associated with treatment of APL, including retinoic acid syn- 
drome, increase the complexity of the treatment. Therefore, timely diagnosis and treatment to avoid complications and related deaths are particularly important. In this report, we present a case of a 28 -year female who was diagnosed with acute promyelocytic leukemia in her second trimester of pregnancy and successfully treated in the First Affiliated Hospital of Dali University. We will report and discuss the patient's medical history, clinical data, treatment methods and results, in order to improve the clinician's understanding of pregnancy with acute promyelocytic leukemia, and improve the outcome of such patients.

\section{Case Report}

A 28 years G1P0 female in her 28 + weeks of gestation, presented to our hospital with complaints of skin freckles on the whole body for half a month and gum bleeding for about 10 days. She was found to be mildly anemic with bleeding gums and slightly cleft lip. An ecchymosis of about $6^{\star} 7 \mathrm{~cm}$ size was visible on the posterior part of the right lower limb. Multiple ecchymoses of varying sizes, biggest being $1^{\star} 2 \mathrm{~cm}$ size, were also visible on the anterior part of the lower limb and upper limbs. There was no lymphadenopathy, no tenderness in the sternum. Respiratory and Cardiovascular examinations were normal. An abdominal examination was also normal except for distended abdomen suggestive of 28 weeks of pregnancy. Baseline laboratory investigations revealed pancytopenia with white blood cell (WBC) count of $1.44^{*} 10^{9} / \mathrm{L}$ (Normal value range $(4-10)^{\star} 10^{9} / \mathrm{L}$ ), neutrophil (NEUT) of $0.58^{\star} 10^{9} / \mathrm{L}$, red blood cell (RBC) count of $2.76^{\star} 10^{12} / \mathrm{L}$ (Normal value range $\left.(3.5-5)^{\star} 10^{12} / \mathrm{L}\right)$, hemoglobin (HGB) of $83 \mathrm{~g} / \mathrm{L}(110-150$ $\mathrm{g} / \mathrm{L})$, platelet $(\mathrm{PLT})$ of $25^{\star} 10^{9} / \mathrm{L}\left((100-300)^{\star} 10^{9} / \mathrm{L}\right)$, and naive cells 0.35 . Coagulation function revealed PT 13.6 seconds (Normal value range 10.5 - 14.5 seconds), APTT 29.8 seconds (Normal value range 31 - 44 seconds), TT 22.9 seconds (Normal value range 14 - 20 seconds), FIB $0.5 \mathrm{~g} / \mathrm{L}$ (Normal value range 2 - $4 \mathrm{~g} / \mathrm{L}$ ), D-2 polymer $5.81 \mathrm{ug} / \mathrm{ml}$ (Normal value range 0 - $500 \mathrm{ug} / \mathrm{ml}$ ). Bone marrow examination revealed extremely active bone marrow hyperplasia with about 35\% abnormal promyelocytes. Bone marrow pathology suggestion: acute myeloid leukemia, tilt M3, bone marrow report picture as shown below (Figure 1 and Figure 2). She was admitted with the diagnosis of acute promyelocytic leukemia with intrauterine pregnancy $28+$ weeks with coagulation dysfunction. The decision to continue the pregnancy with leukemia treatment was made as per the wishes of the patient and the family after active communication regarding the risk of mortality and fetal teratogenicity. In a bid to induce differentiation, she was treated with $20 \mathrm{mg}$ all-tans-retinoic acid (ATRA) twice a day starting on the day of admission. She was also managed with infusion of suspended red blood cells to correct anemia along with infusion of platelet and cryoprecipitate to improve coagulation function. Dynamic fetal heart rate monitoring was carried out throughout this period. Fusion gene investigation detected PML/RARA (bcr1) fusion gene following which "arsenic trioxide (ATO) $8 \mathrm{mg}$ once a day" chemotherapy was added. During this period the patient developed 

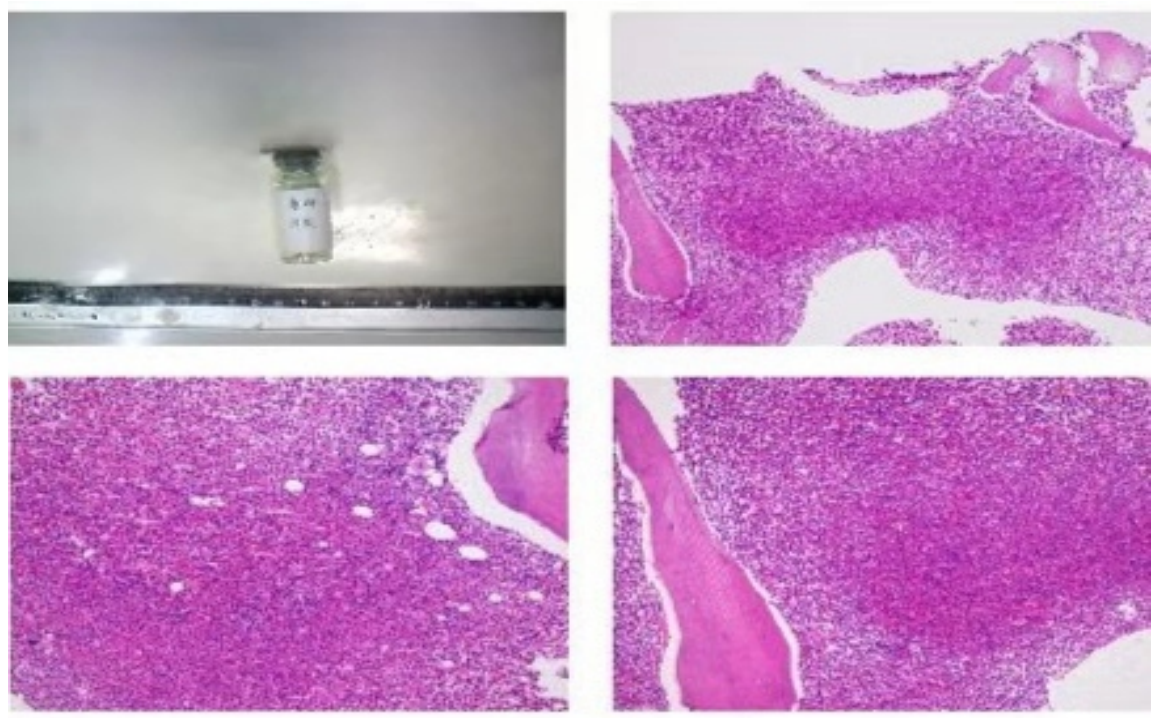

Figure 1. Suggestion: Myeloid hyperplasia is extremely active, only a small number of erythroid and megakaryocytes [CD235a (+erythroid), CD61 (+megakaryosystem)]; monomorphic abnormalities of promyelocytic cells diffuse hyperplasia, IHC showed: MPO (+granule), CD68 (+granule), Ki $+67(+5 \%)$, CD34 (-). Special staining results of bone marrow showed: collagen fiber $(-)$, reticular fiber (MF-1 grade).

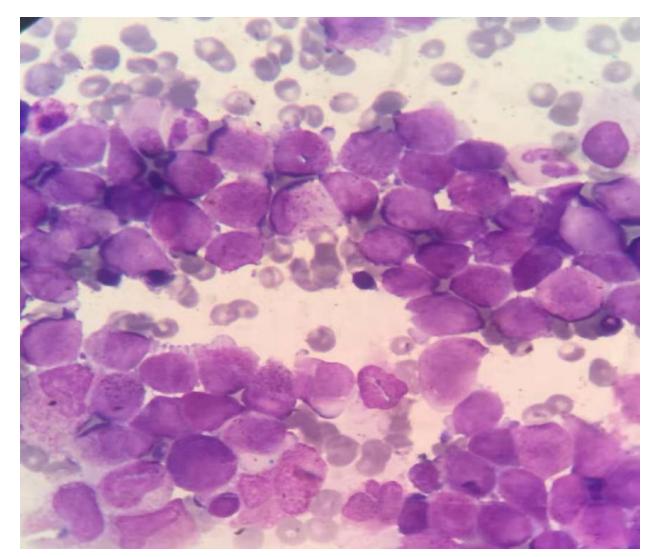

Figure 2. Acute promyelocytic leukemia in peripheral blood smear. Abnormal promyelocytes showed "Austenite body" nuclei and abundant cytoplasmic granules.

lower limb edema and chest tightness. Blood routine investigation (about 2 weeks following admission) during this time was as follows: WBC $26.26^{\star} 10^{9} / \mathrm{L}$, RBC $2.43^{*} 10^{12} / \mathrm{L}, \mathrm{HGB} 74 \mathrm{~g} / \mathrm{L}$, PLT $59^{*} 10^{9} / \mathrm{L}$, naive cells 0.34 . Considering the retinoic acid syndrome, "daunorubicin $40 \mathrm{mg}$, dexamethasone $10 \mathrm{mg}$, furosemide $20 \mathrm{mg}$ " was added to the treatment and the dose of ATRA was adjusted to $20 \mathrm{mg}$ once a day. However, the chest tightness still persisted even after adjusting the treatment plan. Thus, ATRA and ATO were discontinued. Her symptoms relieved after 2 days and the dose of ATRA was adjusted again to $20 \mathrm{mg}$ once a day. Following consultation with obstetrician and request of the patient and her family, it was decided to continue the pregnancy as far as possible. On May 27th, the obstetrics color ultrasound: 1) Intrauterine single live birth, the size of the 
fetus is equivalent to about 32 weeks of pregnancy +, head position; 2) Amniotic fluid is less than the sound image. Initial screening for six fatal malformations: no brain, severe brain swelling, severe open spina bifida, single-chamber heart, severe chest and abdominal wall defects, visceral eversion, fatal cartilage hypoplasia and other abnormalities. About 1 month after admission, the blood routine was reviewed and it revealed WBC $2.67^{\star} 10^{-9} / \mathrm{L}, \mathrm{RBC} 2.47^{\star} 10^{-12} / \mathrm{L}, \mathrm{HGB} 82 \mathrm{~g} / \mathrm{L}$ and PLT258 $10^{-9} / \mathrm{L}$. The coagulation function had returned to normal. Repeat bone marrow report suggested that she had achieved complete remission. The patient was regularly examined during the entire chemotherapy period and no abnormalities were observed in the fetus. Regular examination of blood routines and abnormal examinations outside the hospital was performed. At $39+5$ weeks of pregnancy, she delivered a healthy $3.05 \mathrm{~kg}$ male baby with normal delivery. Apgar scored 1 minute 5 minutes, 5 minutes 9 minutes, 10 minutes 10 minutes. No abnormalities were found in the examination. Blood routine: WBC $12.99^{*} 10^{-9} / \mathrm{L}$, NEUT\% $73.3 \%$, LYMP\% $18.7 \%$, MONO $\% 4.2 \%$, RBC $5.7^{\star} 10^{-12} / \mathrm{L}$, HGB $222 \mathrm{~g} / \mathrm{L}$, PLT $118^{\star} 10^{-9} / \mathrm{L}$, RET\% 5.08\%, late RBC\% $10 \%$, liver function: BI 66.7 umol/L, IBI 56.2 umol/L. Myocardialzymogram showed: CK 862 U/L (Normal value range 24 - $195 \mathrm{U} / \mathrm{L}$ ), CK-MB $94 \mathrm{U} / \mathrm{L}$ (Normal value range 0 - 24 $\mathrm{U} / \mathrm{L}$ ); no abnormalities in cardiac color Doppler ultrasound, comprehensive consideration of the presence of 1) mild asphyxia;2) myocardial damage, staying in neonatology, after symptomatic treatment, improved and discharged. The baby had no congenital abnormalities. The delivery was smooth and there were no complications. After the delivery, the patient did not continue hospitalization and she was followed up by telephone. She had no complaints and both she and the child are doing well.

\section{Discussion}

\subsection{General Treatment of APL in Adults}

At present, treatment of adult APL is divided into two parts: induction therapy and post-remission treatment. Induction therapy is based on the dual induction of ATRA + ATO, for a total of about 4 weeks. An anthracycline or cytarabine and hydroxyurea may be added as appropriate. After remission is achieved, treatment is divided into consolidation therapy and maintenance therapy. Consolidation therapy mainly consists of the use of ATRA + ATO combination therapy. The latest guidelines recommend the use of ATRA + ATO or compound Huang Qi tablets for a total of about 7 months. Maintenance therapy is critical to improving the cure rate in patients with APL. The current recommendation for maintenance treatment regimen is ATRA combined with 6-mercaptopurine and methotrexate for a total of 2 years [3]. The above treatment regimen is seen to be effective in more than $80 \%$ of patients [4].

\subsection{Adverse Effects of Chemotherapy in APL}

In the past few decades, the use of ATRA and arsenic has made considerable 
progress in the treatment of APL. This has greatly improved the cure rate. As a member of the retinoid family, ATRA is considered to have high teratogenicity. The use of ATRA during early pregnancy may cause fetal retinal damage [5]. Other complications that can result from the use of ATRA during the first trimester of pregnancy include craniofacial alterations, neural tube defects, cardiovascular malformations, thymic aplasia, psychological impairments, and kidney alterations. It is also toxic to the fetal heart during the middle and late pregnancy. So ATRA is not recommended for use in early pregnancy. ATRA can be used in the middle and late pregnancy, but due to its cardiotoxicity, fetal heart rate monitoring should be performed frequently [5]. However, Valappil et al. reviewed 27 cases where ATRA was used during pregnancy. No congenital malformations were reported in the newborn in this study, which provided evidence for the use of ATRA during pregnancy. The author will make a corresponding report on the pregnancy from acute promyelocytic leukemia in pregnancy from 2010 to now, see Table 1 for details. All patients were treated with ATRA-induced differentiation, and some patients were treated with doxorubicin, and none of them had teratogenicity, which further proves the above point. Moreover, the ATRA treatment dose for pregnant patients with APL is about $40-45$ $\mathrm{mg} / \mathrm{m}^{2} /$ day, which is no different from that of non-pregnant APL patients, and the dose of doxorubicin is currently inconclusive. Compared with ATRA, anthracyclines are relatively less toxic and can be used in the first trimester. Among anthracyclines, idarubicin is more likely to cross the placental barrier due to the lipophilicity of anthracyclines. Tetramycin is more suitable for early pregnancy than edababicin. ATO has also been reported to have strong embryo toxicity and

Table 1. Case reports of treatment of acute promyelocytic leukemia during pregnancy.

\begin{tabular}{|c|c|c|c|c|c|}
\hline \multirow{2}{*}{$\begin{array}{l}\text { Mother's } \\
\text { Age }\end{array}$} & \multicolumn{2}{|c|}{ Gestational Age (wks) } & \multirow{2}{*}{ Chemotherapy Regimen } & \multirow{2}{*}{ Fetal Outcome } & \multirow{2}{*}{$\begin{array}{l}\text { Maternal } \\
\text { Outcome }\end{array}$} \\
\hline & At diagnosis & At delivery & & & \\
\hline 27 & 7 & $\begin{array}{l}\text { Termination } \\
\text { of pregnancy }\end{array}$ & $\begin{array}{l}\text { Planed for ATRA, then Termination } \\
\text { of pregnancy, ATRA plus idarubicin }\end{array}$ & $\begin{array}{l}\text { Termination } \\
\text { of pregnancy }\end{array}$ & CR \\
\hline 23 & 26 & 27 & ATRA $45 \mathrm{mg} / \mathrm{m}^{2} /$ day & Death & CR \\
\hline 40 & 26 & 30 & $\begin{array}{c}\text { TRA } 45 \mathrm{mg} / \mathrm{m}^{2} / \text { twice day, idarubicin } \\
12 \mathrm{mg} / \mathrm{m}^{2} / \text { every other day }\end{array}$ & A healthy baby & CR \\
\hline 30 & 34 & 35 & $\begin{array}{l}\text { Not treated during pregnancy, } \\
\text { After pregnancy ATO + ATRA }\end{array}$ & $\begin{array}{c}2450 \mathrm{~g} \\
\text { Preterm healthy }\end{array}$ & CR \\
\hline 30 & $23^{+3}$ & 30 & $\begin{array}{l}\text { ATRA } 80 \mathrm{mg} / \text { day, Idarubicin } \\
(20 \mathrm{mg} / \text { day }) \text { was concomitantly } \\
\text { administered on days } 2,4 \text {, and } 5\end{array}$ & $\begin{array}{l}\text { Fetal death } \\
\text { (Hemorrhage) }\end{array}$ & CR \\
\hline 41 & $24^{+4}$ & 35 & ATRA $45 \mathrm{mg} / \mathrm{m}^{2} /$ day & $\begin{array}{l}2950 \text { g preterm } \\
\text { healthy baby }\end{array}$ & CR \\
\hline $\begin{array}{l}\text { two woman } \\
24^{1 \text { st }} 27^{\text {nd }}\end{array}$ & $24^{1 \mathrm{st}} 34^{2 \mathrm{nd}}$ & $25^{1 \mathrm{st}} 36^{\text {nd }}$ & ATRA $45 \mathrm{mg} / \mathrm{m}^{2} /$ day & $\begin{array}{l}\text { Death }^{1 \text { st }} \text { A healthy } \\
3200 \mathrm{~g} \text { female infant }{ }^{2 n d}\end{array}$ & Death ${ }^{1 s t} \mathrm{CR}^{\text {2nd }}$ \\
\hline 23 & 26 & 26 & ATRA $45 \mathrm{mg} / \mathrm{m}^{2} /$ twice & Death & CR \\
\hline
\end{tabular}


is not recommended for use during the entire pregnancy [6]. At present, there are few reports on its use in pregnant APL. Domestic NiuJunjie et al. [7] reported that ATRA combined with ATO was used to treat APL in late pregnancy. Cesarean section was terminated after 20 days of medication. There were no pregnancy complications. Fetal development was normal during follow-up 13 months. It is suggested that ATO may be safe for late pregnancy, but more clinical data are needed to prove it. In this case, the patient used ATO at a dose of 8 $\mathrm{mg} / \mathrm{day}$, and the fetus did not have malformations. In addition, other chemotherapeutic drugs, such as methotrexate, cytarabine and sputum analogs, may increase the risk of cardiotoxicity and should be avoided in early pregnancy.

Another issue is that is important to take into consideration during treatment with ATRA is retinoic acid syndrome. This is a fatal complication of acute promyelocytic leukemia during induction chemotherapy. It is also known as differentiation syndrome (DS). Patients receiving ATRA for APL are at risk of developing this syndrome within 1 - 3 weeks of initiation of induction chemotherapy. There are differences in the incidence of this syndrome in the literature. A review of recent literature indicates that the incidence rate is probably from $2 \%$ to $27 \%$. Its pathogenesis has not been clearly understood. The currently recognized mechanisms are mainly related to adhesion factors, cytokines and chemokines. There are no definite diagnostic criteria for its diagnostic criteria, but most scholars believe that its clinical manifestations include unexplained fever, weight gain, peripheral edema, dyspnea due to pulmonary interstitial infiltration, pleural and pericardial effusion, intermittent hypotension, acute renal failure, and so on. In recent years, some scholars think that it should also include the following aspects: ocular manifestations (bilateral visual acuity decline), skin lesions (granulosercoma suppurative transformation), ET syndrome (acute febrile neutrophil dermatosis), edematous pancreatitis, bradycardia, pericardial tamponade, and pulmonary hemorrhage, etc.). The occurrence of differentiation syndrome can increase the mortality of APL and seriously affect the therapeutic effect of ATRA. Therefore, early understanding and timely treatment of DS is the best way to prevent its progress and reduce its harm to patients. A review of the relevant literature indicates the following points as now recognized in the management:

1) Dexamethasone: Many studies at home and abroad have found that dexamethasone can effectively reduce the secretion of inflammatory cytokines in alveolar epithelial cells, thereby controlling the occurrence of DS. Therefore, dexamethasone $10-20 \mathrm{mg} / \mathrm{d}$ was applied early, until the symptoms disappeared, which is clearly pointed out in the guidelines at home and abroad. However, there is controversy about whether the prophylactic use of glucocorticoids is needed. Studies have shown that patients with high white blood cell count during diagnosis are at higher risk of developing DS. Such patients can benefit from prophylactic use of glucocorticoids. The development of DS can be prevented by using it which can reduce DS incidence and mortality [8]. 
2) Regarding the reduction or discontinuation of ATRA or ATO, various studies have differing opinions. The author believes that if DS is severe and chemotherapy and glucocorticoids are not able to relieve the symptoms, ATRA and ATO should be stopped to ensure the safety of patients.

3) Add other chemotherapy drugs: At present, hydroxyurea, combined chemotherapy, etc. can be used. Combination chemotherapy includes mostly daunorubicin, nordaxin, homoharringtonine and cytarabine. Most studies have shown that DS can be avoided.

4) Other supportive treatments such as oxygen inhalation, diuretics, blood transfusion, platelet transfusion, mechanical ventilation, and other supportive treatments.

In this case, the patient developed retinoic acid syndrome on the 19th day of initiation of induction therapy. "Daunorubicin $40 \mathrm{mg}$, dexamethasone $10 \mathrm{mg}$, furosemide $20 \mathrm{mg}$ ", was added to the treatment regimen while adjusting the ATRA dose to $20 \mathrm{mg}$ once a day from twice a day. As her symptoms persisted despite adjusting the treatment plan, both ATO and ATRA were discontinued. Her symptoms were relieved after 2 days of discontinuing ATRA and ATO. The clinical manifestations observed and treatment applied in this case are consistent with the literature reports. However, this case raises some serious questions. Was the patient more susceptible to develop DS due to her pregnancy? Is there any reproductive toxicity associated with the use of glucocorticoids? Thus, the treatment of differentiation syndrome induced by use of ATRA induction chemotherapy in APL during pregnancy remains to be further studied.

\section{Management}

Reasonable selection of chemotherapy drugs can avoid fetal congenital malformation to a certain extent, but the risk of abortion, premature birth, low birth weight neonatal, neonatal neutropenia and sepsis will still increase. However, based on the lack of clinical and epidemiological evidence, the choice of chemotherapy drugs and the timing of chemotherapy still remain as clinical challenges. Most scholars recommend immediate termination of pregnancy and active anti-leukemia treatment in early pregnancy. In special circumstances, if APL is diagnosed in early pregnancy and the patient is eager to continue the pregnancy, treatment can be actively supported. Chemotherapy can be started after $8-10$ weeks of pregnancy. The 10th week of pregnancy is the most active period of fetal growth and development. The use of cytotoxic drugs during this period has a $10 \%-20 \%$ risk of teratogenicity and is prone to abnormal embryonic development. However, if diagnosis is made in the middle or later stages of pregnancy, chemotherapy should be started immediately and strived to achieve complete remission within a short period. If the condition is critical, chemotherapy should be started at the same time as symptomatic supportive treatment. When the condition becomes stable, the pregnancy should be terminated. Most scholars believe that pregnancy should be as much as possible after 36 weeks. If prema- 
ture birth occurs, it is recommended to use cortisol appropriately to reduce the labor.

The mode of delivery of APL patients is another area that requires careful assessment of the patient's general condition. Normal delivery is preferable to cesarean section as it has a lower risk of bleeding than cesarean delivery. However, cesarean section is the best choice when patients cannot withstand the pressure of normal vaginal delivery. In patients with stable disease, chemotherapy drugs can be suspended for a short time before labor and can be continued after delivery continue [9] [10]. In patients who are diagnosed later during pregnancy, near the time of delivery, chemotherapy can be delayed after delivery.

Pregnancy with APL is extremely challenging and requires the active participation of patients, family members, hematologists, obstetricians and neonatal doctors in decision-making to achieve good outcomes for both patient and fetus. In this case, the patient was diagnosed with APL at 28 weeks of gestation and immediately achieved hematologic remission after receiving induction chemotherapy with ATRA + arsenic trioxide. After chemotherapy, she gave birth to a healthy male baby at 39 weeks +5 days of pregnancy.

\section{Outlook}

As mentioned above, ATO has also been reported to have some embryonic toxicity and is not recommended for use during pregnancy. However, in this case, ATO was used during pregnancy. Nevertheless, no deformity was seen in the baby after the delivery. Successful delivery of normal infants after ATO treatment has been reported by some other studies. Whether arsenic trioxide has dose-dependent toxicity due to its accumulation in the body or it does not have any toxicity during pregnancy remains to be considered. As previously stated, no extensive studies have been performed regarding this issue. Data are limited to a few studies conducted in populations exposed to arsenic from drinkable water or from working at or living near smelters. At the same time, there is no clear evidence that leukemia cells can pass through the placental barrier. Some scholars believe that because the placental barrier can prevent leukemia cells from entering the fetus, it is rare for leukemia to pass through the placenta to the fetus. But the author believes that it is not possible to come to this conclusion as only a few cases of leukemia in pregnancy have been studied. A lot of extensive research and studies still need to be performed regarding leukemia in the later stages of pregnancy.

\section{Conclusions}

APL during pregnancy is a rare occurrence. The literature and research on the outcomes of these patients are also scarce. As discussed above, both ATRA and ATO have been reported to have embryonic toxicity and are not recommended to be used during pregnancy. Nevertheless, successful outcome has been documented in pregnant females who have been treated with ATRA in some studies. 
The case described in this report was diagnosed with APL in last trimester of pregnancy and there was further complication of differentiation syndrome. Despite receiving the combination of ATRA and ATO and developing differentiation syndrome, she gave birth to a healthy infant with no abnormalities.

Whether the toxicity of ATO is dose-dependent or it does not have any toxicity during pregnancy remains to be considered. No studies have been performed about this and there is still room for more extensive research and placental pathological examination after pregnancy [11].

\section{Conflicts of Interest}

The authors declare no conflicts of interest regarding the publication of this paper.

\section{References}

[1] Aoki, A., Yoneda, N., Yoneda, S., et al. (2011) Massive Postpartum Hemorrhage After Chemotherapy in a Patient with Acute Promyelocytic Leukemia. Journal of Obstetrics and Gynaecology Research, 37, 1759-1763. https://doi.org/10.1111/j.1447-0756.2011.01598.x

[2] O”Donnell, M.R., Tallman, M.S., Abboud, C.N., et al. (2017) Acute Myeloid Leukemia, Version 3.2017, NCCN Clinical Practice Guidelines in Oncology. Journal of the National Comprehensive Cancer Network, 15, 926-957. https://doi.org/10.6004/jnccn.2017.0116

[3] Yang, L., Gong, Y.P., Yang, Y.M., et al. (2010) Successful Treatment of a Case of ATRA and ATO in the Treatment of Relapsed APL with TanshinoneIIA. Journal of Sichuan University (Medical Science Edition), 41, 1065-1067.

[4] Sanz, M.A., Grimwade, D., Tallman, M., et al. (2009) Management of Acute Promyelocytic Leukemia: Recommendations from an Expert Panel on Behalf of the European LeukemiaNet. Blood, 113, 1875-1891. https://doi.org/10.1182/blood-2008-04-150250

[5] Milojkovic, D. and Apperley, J.F. (2014) How I Treat Leukemia during Pregnancy. Blood, 123, 974-984. https://doi.org/10.1182/blood-2013-08-283580

[6] Du, X. (2010) Progress in the Treatment of Pregnancy Complicated with Acute Leukemia. Foreign Journal of Blood Transfusion and Hematology, 33, 511-512.

[7] Niu, J.J., Duan, W.Y., Cui, X.P., et al. (2013) Analysis of Arsenic Trioxide All-Trans Retinoic Acid Chemotherapy in Pregnancy with Acute Promyelocyticleukemia. Journal of Practical Medical Techniques, 20, 197-198.

[8] Rogers, J.E. and Yang, D. (2012) Differentiation Syndrome in Patients with Acute Promyelocyticleukemia. Journal of Oncology Pharmacy Practice, 18, 109-114. https://doi.org/10.1177/1078155211399163

[9] Terada, Y., Shindo, T., Endoh, A., et al. (1997) Fetal Arrhythmia during Treatment of Pregnancy-Associated Acute Promyelocytic Leukemia with All-Trans Retinoic Acid and Favorable Outcome. Leukemia, 11, 454-455. https://doi.org/10.1038/sj.leu.2400595

[10] Xu, W. (2013) Treatment of Acute Promyelocytic Leukemia with Pregnancy. Journal of Clinical Hematology, No. 1, 45-47.

[11] Fei, F., Faye-Petersen, O.M., Vachhani, P., et al. (2019) Acute Promyelocytic Leu- 
kemia during Pregnancy: A Case Report and 10-Year Institutional Review of Hematologic Malignancies during Pregnancy. Pathology-Research and Practice, 215, Article ID: 152672. 